\title{
Erratum: Reinforcement learning in professional basketball players
}

Tal Neiman \& Yonatan Loewenstein

Nature Communications 2:569 doi: 10.1038/ncomms1580 (2011); Published 6 Dec 2011; Updated 15 Jan 2013.

This Article contains typographical errors in Equation (2), in which incorrect characters are displayed. Equation (2) should read:

$$
Q_{i}(t+1)=Q_{i}(t)+\eta_{i} \delta_{i, a(t)}\left(R(t)-Q_{i}(t)\right) \quad i \in\{2,3\}
$$

